\title{
LÉOPOLD DOR Équations caractéristiques de la comptabilité analytique
}

Revue française d'automatique, d'informatique et de recherche opérationnelle. Recherche opérationnelle, tome 3, n V2 (1969), p. 75-105.

<http://www.numdam.org/item?id=RO_1969_3_2_75_0>

(C) AFCET, 1969, tous droits réservés.

L'accès aux archives de la revue « Revue française d'automatique, d'informatique et de recherche opérationnelle. Recherche opérationnelle » implique l'accord avec les conditions générales d'utilisation (http://www.numdam.org/ legal.php). Toute utilisation commerciale ou impression systématique est constitutive d'une infraction pénale. Toute copie ou impression de ce fichier doit contenir la présente mention de copyright.

\section{Numdam}

Article numérisé dans le cadre du programme

Numérisation de documents anciens mathématiques

http://www.numdam.org/ 
R.I.R.O.

(3e année, $\mathrm{N}^{\mathrm{O}} \mathrm{V}-2,1969$, p. $\left.75-106\right)$

\title{
EQUATIONS GARAGTERISTIQUES DE LA GOMPTABILITE ANALYTIQUE
}

\author{
par Léopold Dor
}

\begin{abstract}
Résumé. - En comptabilité analytique, la ventilation des charges sur les produits permet d'écrire les équations d'équilibre ponctuel d'une comptabilité complète.

La fixité de certaines charges conduit à l'optimisation de tendances en comptabilité marginaliste.

Le partage des responsabilités suscite des sous-optimisations locales avec comptabilité des écarts.

Ces analyses distinctes peuvent être reprises dans un même schéma matriciel basé sur la structure additive des coûts, d'où se déduit une gestion par ratio contrôlé.
\end{abstract}

Notre propos résume un essai d'intégration de la comptabilité analytique et de la recherche opérationnelle. On y trouvera donc la séquence classique de toute R.O. : analyse, critique, proposition.

L'analyse sera forcément rapide car les systèmes de comptabilité analytique sont très variés. Elle se veut cependant systématique : à partir des principes caractérisant les comptabilités les plus typiques, nous avons cherché à formuler uniformément le processus suivi et obtenu des équations qui se complètent.

La critique de leurs propriétés et de leur portée suscite des remarques diverses concernant notamment l'usage qui en est fait, engendre un certain scepticisme touchant au mythe de l'optimisation, puis finalement provoque un plaidoyer, peut-être naïf, pour une gestion par ratio contrôlé : la proposition.

Cette proposition est un aboutissement au développement suivant :

1) Principe de ventilation.

2) Matrice de ventilation.

3) Équations fondamentales de production et de consommation.

4) Principe de fixité.

5) Optimisation marginaliste.

Chargé de Cours à l'Université de Liège, Chef de Service à la S.A. Cockerill-Ougrée-Providence. 
6) Principe du partage des responsabilités.

7) Analyse des écarts.

8) Structure additive.

\section{1) PRINCIPE DE VENTLATION}

Lorsque le chef d'entreprise désire obtenir des prix de revient incluant toutes les charges concourant à la réalisation d'une production multiple, soit des prix de revient complets, il doit admettre le principe d'une ventilation, sur chacun des produits, de charges qui ne leur sont cependant pas directement consacrées. Ce principe de ventilation dont l'aspect simplificateur sinon arbitraire apparaît de suite, a reçu des expressions de plus en plus raffinées. Partie d'un coefficient global de frais généraux appliqués à la masse des frais directs, passée ensuite à un coefficient d'imputation pondérée, la comptabilité du prix de revient complet en est arrivée à une méthode par étapes, méthode dite des sections homogènes parce que, à chaque étape, se trouvent des regroupements de charges selon un caractère commun, en relation certaine ou aléatoire avec les quantités de facteurs ou de produits concernant la section.

Cette évolution de la méthode se concrétise par le recours à des clés de répartition des charges sur les sections soit à des critères plus ou moins circonstanciés et plus ou moins objectifs. Le flot des charges se développe alors selon le graphe de ventilation (figure 1)

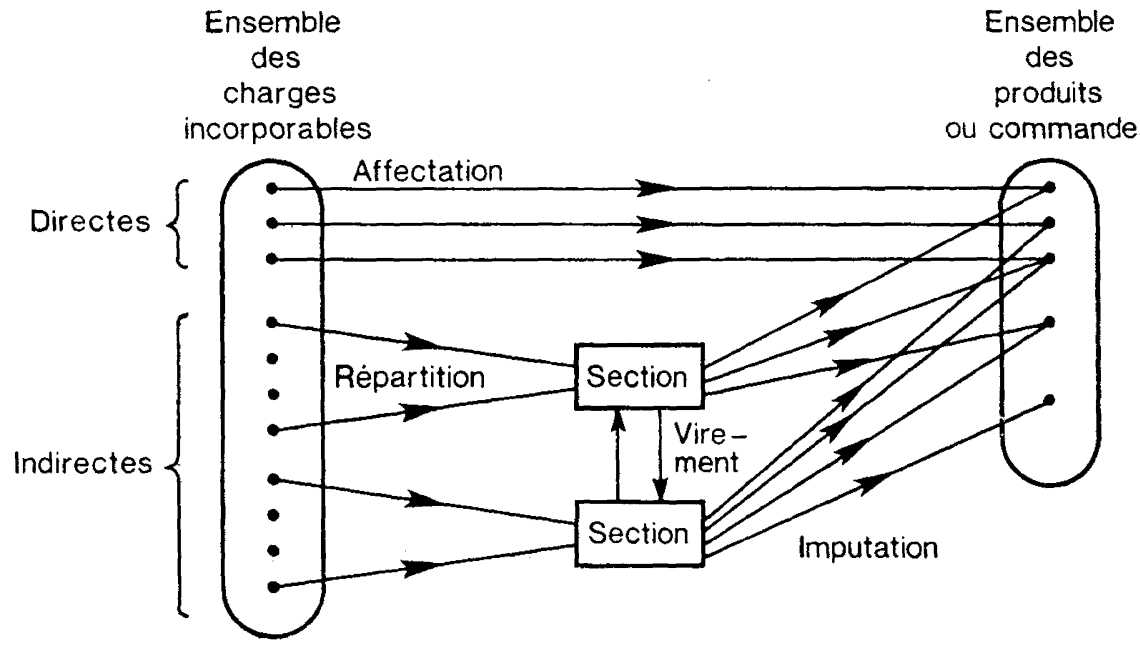

qui dégage :

Figure 1

- l'affectation des charges directes aux produits;

- la répartition des charges indirectes sur les sections transitoires.

Ces charges sont parfois dites semi-directes lorsqu'elles sont supportées dans leur totalité par une seule section transitoire; 
- la sous-répartition ou virement d'une section transitoire sur l'autre. En effet, il est parfois nécessaire de redistribuer les charges entre les sections transitoires, les unes travaillant au profit des autres. Ainsi une section chaudières produit de la vapeur au profit d'une section centrale électrique qui fournira du courant pour actionner les chaudières. Le virement d'une section transitoire sur l'autre correspond à l'existence d'un circuit sur le graphe de ventilation;

- l'imputation des frais des sections transitoires aux sections de production.

En pratique extra-comptable, ces opérations précédentes se font sur un tableau de répartition, tableau à double entrée avec en lignes les diverses charges, en colonnes les diverses sections de production et de transit; le tableau en donne un exemple simple (inspiré de ParenteauCharmont, p. 51).

La $1^{\text {re }}$ partie du tableau ventile les charges par sections. La $2^{\mathrm{e}}$ partie ventile les totaux des sections transitoires en commençant ici par l'entretien, étant entendu que l'entretien doit également supporter une part

TABLEAU a. - Tableau de répartition des charges par sections homogènes et calcul du prix de revient complet historique

\begin{tabular}{|c|c|c|c|c|c|c|c|}
\hline \multirow[b]{2}{*}{ Nature } & \multicolumn{4}{|c|}{ SEGTIONS DE PRODUCTION } & \multicolumn{2}{|c|}{ SECT. TRANSITOIRES } & \multirow{2}{*}{ Tотах } \\
\hline & $X$ & $\boldsymbol{Y}$ & $Z$ & $\Sigma$ & $\begin{array}{l}\text { Services } \\
\text { entret. }\end{array}$ & $\begin{array}{c}\text { Services } \\
\text { administ. }\end{array}$ & \\
\hline Matières premières & $300 \mathrm{~F}$ & $500 \mathrm{~F}$ & $650 \mathrm{~F}$ & $1450 \mathrm{~F}$ & $50 \mathrm{~F}$ & $50 \mathrm{~F}$ & $1550 \mathrm{~F}$ \\
\hline Salaires. ...... & 350 & 200 & 900 & 1450 & 100 & 150 & 1700 \\
\hline Appointements $\ldots \ldots \ldots$ & 200 & 100 & 150 & 450 & 100 & 150 & 700 \\
\hline Énergie. $\ldots \ldots \ldots \ldots \ldots$ & 50 & 250 & 100 & 400 & 50 & - & 450 \\
\hline Mat. consommation $\ldots \ldots$ & 50 & 200 & 100 & 350 & 50 & 50 & 450 \\
\hline \multirow[t]{2}{*}{ Amortissement $\ldots \ldots \ldots$} & 50 & 150 & 100 & 300 & 50 & 40 & 390 \\
\hline & 1000 & 1400 & 2000 & 4400 & \multirow{7}{*}{$\begin{array}{c}400 \\
\leftarrow \\
\text { Imputé } \\
\text { par } \\
\text { quantité } \\
\text { produite }\end{array}$} & 440 & 5240 \\
\hline \multirow[t]{2}{*}{ Services entretien....... } & 100 & 100 & 200 & 400 & & \multirow{6}{*}{$\begin{array}{l}\leftarrow \text { Imputé } \\
\text { par } \\
\text { totaux de } \\
\text { section }\end{array}$} & \\
\hline & 1100 & 1500 & 2200 & 4800 & & & \\
\hline Services administratifs. . . & 100 & 140 & 200 & 440 & & & \\
\hline Totaux complets. ....... & 1200 & 1640 & 2400 & 5240 & & & \\
\hline Nombre d'unités d'œuvre. & $100 X$ & $100 Y$ & $200 Z$ & & & & \\
\hline Prix de revient complet. . & $12 \mathrm{~F}$ & $16,4 \mathrm{~F}$ & $12 \mathrm{~F}$ & & & & \\
\hline
\end{tabular}


des charges d'administration. Compte tenu de la clé adoptée, le procédé est commode. Mais le procédé inverse serait préférable dans d'autres cas. (Voir par exemple R. Henon, pp. 71-73.) $X, Y, Z$ désignent des produits ou des commandes.

\section{2) MATRICE DE VENTILATION}

\section{Cas simples}

Si $g=\left(g_{i}\right)>0$ est le vecteur des $n$ charges $i$ supposées indépendantes et exprimées dans une même unité et $q=\left(q_{j}\right)>0$ le vecteur du niveau physique des $m$ produits $j$ supposés indépendants, le problème de la comptabilité analytique des prix de revient complets (historiques) est celui de la détermination des composantes de $c=\left(c_{j}\right)>0$ vecteur des prix de revient complets des $m$ produits $j$, prix exprimés dans la même unité que $g$, soit celui de la détermination de $\Gamma=\left(\Gamma_{j}\right)>0$ vecteur des coûts totaux des $m$ produits $j$

où

soit

$$
\left.\begin{array}{l}
\Gamma_{j}=c_{j} q_{j} \quad \forall j=1, \ldots, m \\
\Gamma=\operatorname{Diag} q \cdot c=\operatorname{Diag} c \cdot q
\end{array}\right\}
$$

Diag $x$ étant la matrice diagonale dont les composantes non diagonale principale sont nulles, et celles de la diagonale principale égales aux composantes du vecteur $x$.

Le coût total de l'ensemble de la production $C_{T}$ est soit la somme des $n$ charges soit la somme des coûts totaux des $m$ produits

$$
\begin{aligned}
C_{T} & =e^{*} \cdot g=\Gamma^{*} \cdot e & \\
& =c^{*} \cdot q & \text { d'après (1) }
\end{aligned}
$$

où $e$ est le vecteur dont chaque composante est l'unité

* indique la transposition

$x$ étant vecteur-colonne, $x^{*}$ est le vecteur-ligne correspondant

(2) est l'équation de la balance comptable des coûts et des charges.

Si la production est simple, c'est-à-dire $m=1$, le problème de la comptabilité analytique est de solution immédiate puisque (2) se réduit à

$$
g=c q \quad \text { d'où } \quad c=\frac{g}{q}
$$

Si le système de production est simple, comme dans un système d'échanges équilibrés d'input-output, toutes les charges $g$ sont affectées au produit.

Pour distinguer ce cas, remplaçons $g=\left(g_{i}\right)$ par $a=\left(a_{i}\right) \geqq 0$ dont les composantes $a_{i j}$ sont les charges de nature $i$ affectées au produit $j$, 
mais aussi les coûts du produit $j$ provenant directement de la charge $i$, de telle sorte qu'on écrira

pour les charges affectées pour les coûts totaux directs

Si

$$
a_{i}=\sum_{j=1}^{m} a_{i j}
$$

$$
a_{i j}=\alpha_{i j} q_{j}
$$

$\alpha_{i j}>0$ étant le coefficient d'affectation de la charge $i$ par unité de produit $j$,

$A=\left[\alpha_{i j}\right] \geqq 0$ la matrice des coefficients d'affectation de format $n \times m$, et $\gamma=\left(\gamma_{j}\right)>0$ le vecteur des prix de revient directs

(3) s'écrira :

$$
\begin{aligned}
a_{i}=\sum_{j} \alpha_{i j} q_{j} & =q_{j} \sum_{i} \alpha_{i j} \\
& =q_{j} \gamma_{j} \\
\text { où } \gamma_{j} & =\sum_{i=1}^{n} \alpha_{i j} \geqslant 0 \\
\text { est le prix de revient } & \text { direct du produit } j \\
\text { soit } a=A \cdot q \quad & d^{*}=\gamma^{*} \cdot \operatorname{Diag} q \\
\text { où } \gamma^{*} & =e^{*} \cdot A
\end{aligned}
$$

satisfaisant à la balance (2), comme on le vérifie aisément.

Les relations (5) s'obtiennent dualement en considérant respectivement les lignes (pour a) et les colonnes (pour $d$ ) sur le bloc d'affectation, soit

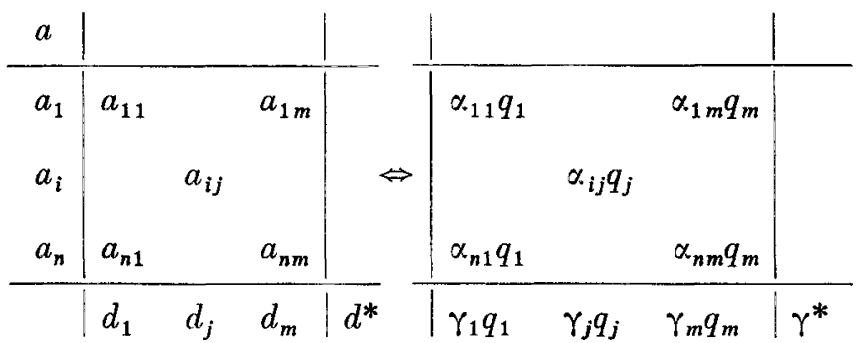

L'équivalence des deux expressions est conditionnée par (4).

La matrice $A$ synthétise ainsi la correspondance entre le point $a$ de l'orthant positif $\mathcal{R}_{+}^{n}$ et le point $q$ de $\mathcal{R}_{+}^{m}$, telle qu'elle s'exprime sur le bloc d'affectation.

A chaque équilibre ponctuel entre $a$ et $q$, correspondra un prix de revient direct $\gamma$. 
L. DOR

On appelle modèle linéaire de production, le cas particulier pour lequel chaque $\alpha_{i j}$ est constant quel que soit $q$, il implique la constance de $\gamma$.

\section{Objet de la ventilation.}

Lorsque la ventilation des charges ne se réduit pas à une affectation, la méthode des sections homogènes admet que :

- la charge de nature $i$ peut être décomposée en deux parties, soit

avec

$$
\left.\begin{array}{ll}
g_{i}=a_{i}+b_{i} & \forall i=1, \ldots, n \\
a_{i}=\sum_{j=1}^{m} a_{i j} & \text { et } \quad b_{i}=\sum_{h=1}^{s} b_{i h}
\end{array}\right\}
$$

où $a_{i}$ est comme ci-dessus le montant de charge $i$ affectée

$b_{i}$ est le montant de charge $i$ non affectée aux produits et $b_{i h}$ la part de $b_{i}$ répartie sur la section transitoire $h$ soit

- le coût total du produit $j$ peut être décomposé en deux parties,

avec $\quad d_{j}=\sum_{i=1}^{n} a_{i j} \quad$ et $\left.\quad k_{j}=\sum_{f=1}^{s} k_{f j}\right\}$

où $d_{j}$ est comme ci-dessus le coût total direct du produit $j$

$k_{j}$ le coût total indirect du produit $j$

et $k_{f j}$ les frais de la section transitoire $f$ imputés à la production du produit $j$.

Le problème à résoudre est alors celui de la détermination du vecteur $\left(k_{j}\right)$ à partir du vecteur $\left(b_{i}\right)$, soit plus précisément celui du passage $\mathrm{du}$ bloc de répartition $\left[b_{i h}\right]$ au bloc d'imputation $\left[k_{f j}\right]$ dans le tableau de répartition des charges par sections homogènes et de calcul des prix de revient complets historiques composé de quatre blocs caractéristiques :

Frais par section

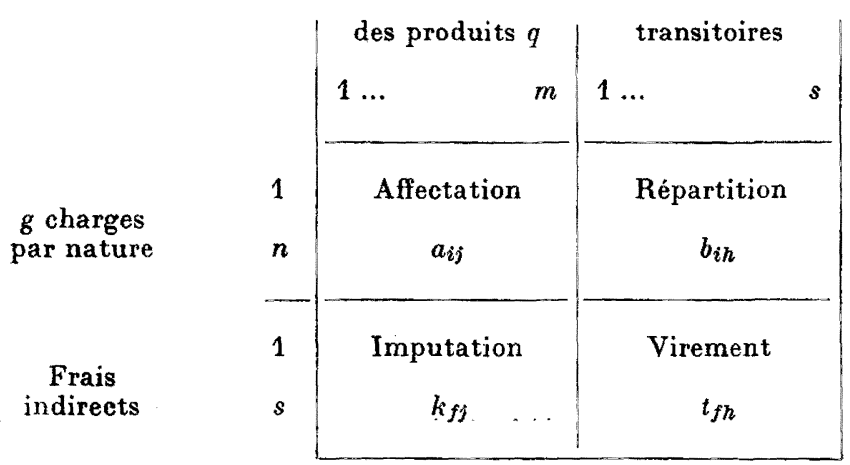


La procédure comptable basée sur le principe de ventilation résoud le problème en trois étapes successives : toires ;

- la répartition des charges non affectées sur des sections transi-

- le redressement des frais de ces sections par virement;

- l'imputation aux produits des frais virés.

Nous allons voir que chacune de ces trois étapes est caractérisée par une matrice, dont le produit fournit la solution recherchée.

\section{Equation de répartition}

Le bloc de répartition découle de l'adoption de clés de répartition et fait passer du vecteur des charges non affectées $b=\left(b_{i}\right) \geqslant 0$ au vecteur des frais transitoires $t=\left(t_{h}\right) \geqq 0$ en admettant

$$
b_{i h}=b_{i} \beta_{i h} \quad \text { avec } \quad \sum_{h=1}^{s} \beta_{i h}=1
$$

de telle sorte que

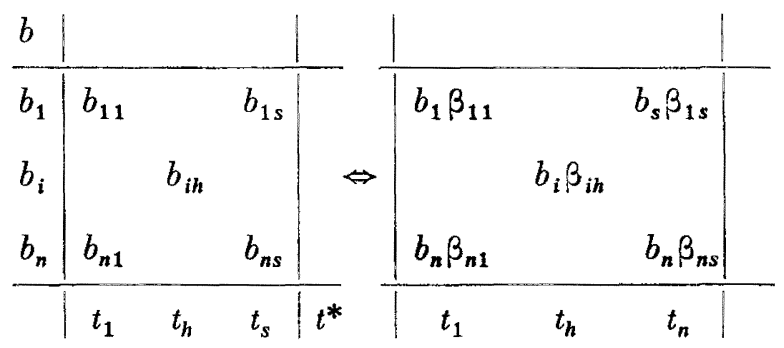

Adopter des clés de répartition revient ainsi à adopter une $B=\left[\beta_{i h}\right]$ matrice des coefficients de répartition $\beta_{i h}$ de telle sorte que les totaux par lignes et par colonnes sur le bloc de répartition conduisent aux équations

soit

$$
\begin{aligned}
& b_{i}=\sum_{h=1}^{s} b_{i h} \quad \text { avec } \quad t_{h}=\sum_{i=1}^{n} b_{i h} \\
& =b_{i} \sum_{h} \beta_{i h} \\
& =\sum_{i=1}^{n} b_{i} \beta_{t h}
\end{aligned}
$$

$B$, matrice de format $n \times s$, est du type markovien si en outre $0 \leqq \beta_{i h} \leqq 1$, disposition coutumière.

\section{Equations de virement}

Le bloc virement fait passer du vecteur $t$ des frais transitoires à un vecteur des frais à imputer $u=\left(u_{f}\right) \supseteqq 0$. Il a pour objet d'éliminer l'inter- 
dépendance des sections transitoires, éventuellement acceptée dans la répartition pour des raisons pratiques.

En écrivant $t_{f h}=\mu_{f h} t_{h}$ avec

de telle sorte que

$$
\sum_{f=1}^{s} \mu_{f h}=1
$$

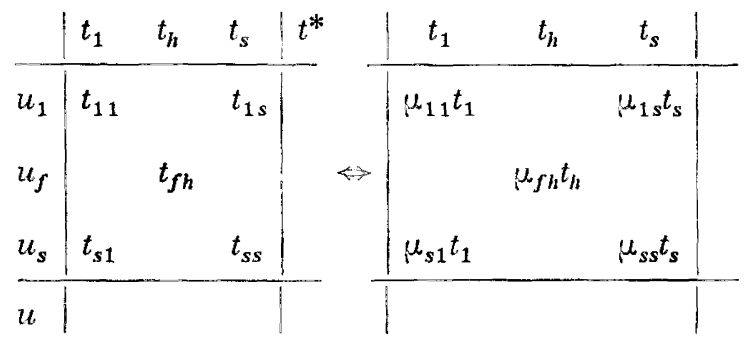

avec $M=\left[\mu_{f h}\right]$ matrice des coefficients de virement $\mu_{f h}$ matrice carrée de format $s \times s$, les totaux par lignes et par colonnes conduisent aux équations

soit

$$
\begin{aligned}
& u_{f}=\sum_{h=1}^{s} t_{f h} \quad \text { avec } \quad t_{h}=\sum_{f=1}^{s} t_{f h} \\
& =\sum_{h=1}^{s} \mu_{f h} t_{h} \\
& =t_{h} \sum_{f} \mu_{f h} \\
& u=M \cdot t \\
& e^{*}=e^{*} \cdot M
\end{aligned}
$$

Si $t$ est viré tel quel, $M=I$.

Si $0 \leqq \mu_{f h} \leqq 1, M$ est markovienne.

\section{Equations d'imputation}

Le bloc imputation fait passer du vecteur $u$ des frais à imputer au vecteur des coûts totaux indirects $k=\left(k_{f}\right) \geqq 0$ en admettant

$$
k_{f j}=u_{f} \eta_{f j} \quad \text { avec } \quad \sum_{j=1}^{m} \eta_{f j}=1
$$

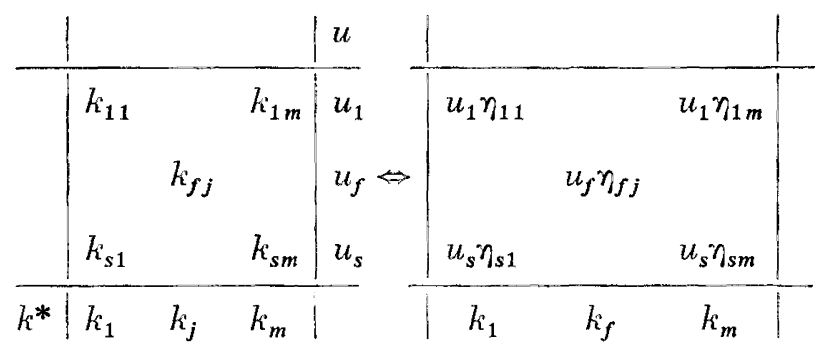


Adopter des clés d'imputation revient à adopter une $N=\left[\eta_{f j}\right]$ matrice des coefficients d'imputation $\eta_{f j}$ de format $s \times m$, de telle sorte que les totaux par lignes et par colonnes sur le bloc d'imputation conduisent aux équations

soit

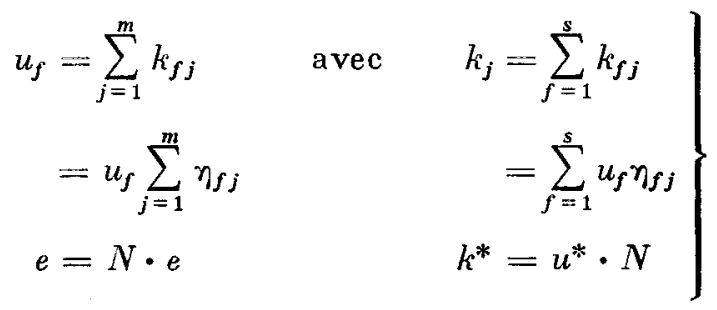

\section{Matrice de ventilation du coût total}

A partir des équations (13), (11) et (9), on obtient la solution du passage du vecteur $b$, des charges non affectées, au vecteur $k$ des coûts indirects, soit

où

$$
\begin{aligned}
& k=N^{*} \cdot M \cdot B^{*} \cdot b \\
& k=T^{*} \cdot b \\
& T=B \cdot M^{*} \cdot N
\end{aligned}
$$

avec

matrice des coefficients de ventilation des charges indirectes de format $n \times m$, comme la matrice des coefficients d'affectation $A$.

De plus, $T$ est markovienne car selon (9), (11) et (13),

$$
T \cdot e=e
$$

et chacune de ses composantes est non négative puisqu'elle constitue un coefficient d'apport des charges indirectes aux charges affectées. Ainsi, à partir de (7), on écrira l'équation vectorielle du coût total

$$
\Gamma=\operatorname{Diag} q \cdot A^{*} \cdot e+T^{*} \cdot b
$$

d'où l'on déduira l'équation vectorielle du prix de revient complet

$$
\begin{aligned}
& c=\operatorname{Diag} \frac{1}{q} \cdot \Gamma \\
& c=A^{*} \cdot e+\operatorname{Diag} \frac{1}{q} \cdot T^{*} \cdot b
\end{aligned}
$$

solution recherchée, somme d'une composante directe provenant de la matrice des coefficients d'affectation et d'une composante indirecte tributaire des clés de ventilation regroupées en $T$. 
Toutefois, $A$ et $T$, qui sont de même format $n \times m$, ventilent respectivement les charges affectées par unité de produit et les unités de charges non affectées sur le vecteur de production.

On rétablit l'homogénéité de ventilation de toutes les charges sur le vecteur de production en considérant

$$
G=A \cdot \operatorname{Diag} q+\operatorname{Diag} b \cdot T
$$

qui apparaît ainsi comme la matrice de ventilation des $n$ charges sur les $m$ produits ou matrice de ventilation du coût total, soit la matrice dont la somme des lignes donne le vecteur des charges, et celle des colonnes le vecteur des coûts par produit, c'est-à-dire

$$
\Gamma^{*}=e^{*} \cdot G \quad \text { et } \quad g=V \cdot e
$$

avec $G=\left[g_{i j}\right] \geqslant 0$

$g_{i j}$ sera la part totale de la charge $g_{i}$ ventilée sur la quantité de produit $q_{j}$, pour l'équilibre établi entre $g$ et $q$.

\section{3) EQUATIONS FONDAMENTALES DE PRODUCTION ET DE CONSOMMATION}

\section{Matrice des coefficients techniques de production}

L'analyse précédente a été menée sans tenir compte du fait que chaque charge $g_{i}$ est le produit d'une quantité de facteur $f_{i}$ et d'un prix $h_{i}$, celui de l'unité de ce facteur, c'est-à-dire que $g_{i}=f_{i} h_{i}$.

Si $\quad f=\left(f_{i}\right)>0$ est le vecteur des quantités factorielles et $\quad h=\left(h_{i}\right)>0$ celui des prix factoriels

on peut écrire

$$
g=\operatorname{Diag} h \cdot f=h \cdot \operatorname{Diag} f
$$

En divisant chaque ligne $i$ de $G$ par $h_{i}$, c'est-à-dire en considérant

$$
\operatorname{Diag} \frac{1}{h} \cdot G
$$

on neutralise l'effet des prix factoriels et la matrice du coût total devient celle du coût factoriel total.

Si on divise ensuite chaque colonne $j$ par la quantité produite $q_{j}$, on obtient :

$$
\left.\begin{array}{rl}
W & =\operatorname{Diag} \frac{1}{h} \cdot G \cdot \operatorname{Diag} \frac{1}{q} \\
\text { soit } \quad W & =\left[\omega_{i j}\right]>0 \quad \text { avec } \quad \omega_{i j}=\frac{g_{i j}}{h_{i} q_{j}}
\end{array}\right\}
$$


Matrice des coefficients techniques de production après ventilation, de format $n \times m$

$w_{i j}$ étant la quantité de facteur $i$ estimée après ventilation, nécessaire à la production d'une unité de produit $j$, pour la production globale $q$ et la consommation globale $f$.

$W$ peut aussi être dite matrice de Walras.

La matrice de ventilation du coût factoriel total peut donc s'écrire

$$
\operatorname{Diag} \frac{1}{h} \cdot G=W \cdot \operatorname{Diag} q
$$

tandis que la somme de ses lignes retrouve le vecteur des facteurs, d'où l'équation de consommation factorielle

$$
f=W \cdot \operatorname{Diag} q \cdot e
$$

c'est-à-dire $\quad$ avec $W \geqq 0$ et $q>0$

En divisant d'abord chaque colonne de $G$ par $q$, c'est-à-dire en considérant $G \cdot \operatorname{Diag} \frac{1}{q}$, on obtient la matrice de ventilation des prix de revient, avec

$$
G \cdot \operatorname{Diag} \frac{1}{q}=\operatorname{Diag} h \cdot W
$$

dont la somme des colonnes retrouve (2.17) sous la forme

$$
\begin{aligned}
& c^{*}=e^{*} \cdot \operatorname{Diag} h \cdot W \\
& c=W^{*} \cdot h \quad \text { avec } \quad W^{*} \geqq 0 \text { et } q>0
\end{aligned}
$$

équation matricielle du prix de resient associée à l'équation de consommation factorielle.

En résumé, étant donné la ventilation de la consommation factorielle totale $f$ sur la production $q$ par une matrice de coefficients techniques $W$, soit (2), le prix de revient complet $c$ est déterminé à partir des prix factoriels imputés selon la transposée de la matrice des coefficients techniques $W^{*}$, soit $(5)$.

Remarquons enfin que le coût total de la production (2.2) est aussi

$$
C_{T}=e^{*} \cdot \mathrm{g}=\Gamma^{*} \cdot e=h^{*} \cdot W \cdot q
$$




\section{Matrice des rendements techniques}

En procédant comme ci-dessus, mais en posant

$$
\left.\begin{array}{rl}
R & =\operatorname{Diag} \frac{1}{f} \cdot G \cdot \operatorname{Diag} \frac{1}{c} \\
& =\left[r_{i j}\right] \geqslant 0 \quad \text { avec } \quad r_{i j}=\frac{g_{i i}}{f_{i} \cdot c_{j}}
\end{array}\right\}
$$

on obtient la matrice des rendements techniques après ventilation de format $n \times m, r_{i j}$ étant la quantité de produit $j$, estimée après ventilation, obtenue à partir de l'unité de facteur $i$ pour la production globale $q$ et la consommation globale $f$. Comme ci-dessus, on écrit :

$$
G \cdot \operatorname{Diag} \frac{1}{c}=\operatorname{Diag} f \cdot R
$$

matrice de ventilation de la production qui permet l'équation de production

$$
q=R^{*} \cdot f \quad \text { avec } \quad R^{*} \geqq 0 \text { et } f>0
$$

tandis que

$$
\operatorname{Diag} \frac{1}{f} \cdot G=R \cdot \operatorname{Diag} c
$$

matrice de ventilation des prix factoriels engendre :

$$
h=R \cdot c \quad \text { avec } \quad R \geqq 0 \text { et } q>0
$$

équation matricielle du prix factoriel associée à l'équation de la production.

De même, le coût total de la production s'écrit

$$
C_{T}=c^{*} \cdot q=f^{*} \cdot h=f^{*} \cdot R \cdot C .
$$

Les matrices $W$ et $R$ déduites des $G$ sont liées, ainsi

$$
W=\operatorname{Diag} \frac{f}{h} \cdot R \cdot \operatorname{Diag} \frac{c}{q}
$$

En remplaçant $W$ par cette valeur

$$
\begin{array}{ll}
\text { dans } f=W \cdot q & \text { on retrouve } h=R \cdot c \\
\text { dans } c=W^{*} \cdot h & \text { on retrouve } q=R^{*} \cdot f \\
\text { dans } h^{*} \cdot W \cdot q & \text { on retrouve } f^{*} \cdot R \cdot c
\end{array}
$$

Les deux systèmes : l'un de consommation et l'autre de production, caractérisés respectivement par la matrice des coefficients techniques et par celle des rendements, sont donc rendus équivalents par (13). 
$W$ est ainsi une transformation sur l'espace des biens, soit $\mathcal{R}_{+}^{n+m}=\{f, q\}$, et la transposée est une transformation sur l'espace des valeurs, soit $\mathcal{R}_{+}^{m+n}\{c, h\}$.

Néanmoins, de l'existence des deux systèmes d'équations on peut déduire :

$$
\left.\left.\begin{array}{rl}
f & =W \cdot R^{*} \cdot f \\
h^{*} & =h^{*} \cdot W \cdot R^{*}
\end{array}\right\} \text { I } \quad \text { et } \quad \begin{array}{rl}
q & =R^{*} \cdot W \cdot q \\
c^{*} & =c^{*} \cdot R^{*} \cdot W
\end{array}\right\} \text { II }
$$

montrant que $f$ et $h^{*}$ sont les vecteurs propres respectivement à droite et à gauche correspondant à la valeur propre un de

$$
\left.\begin{array}{rl}
W \cdot R^{*} & =\operatorname{Diag} \frac{f}{h} \cdot R \cdot \operatorname{Diag} \frac{c}{q} \cdot R^{*}, \text { de format } n \times n \\
& =W \cdot \operatorname{Diag} \frac{q}{c} \cdot W^{*} \cdot \operatorname{Diag} \frac{h}{f} \\
& =\operatorname{Diag} \frac{1}{h} \cdot G \cdot \operatorname{Diag} \frac{1}{\Gamma} \cdot G^{*} \cdot \operatorname{Diag} \frac{1}{f}
\end{array}\right\}
$$

comme on le vérifie aisément à l'aide de (2.9)

tandis que $q$ et $c^{*}$ le sont pour :

$$
\left.\begin{array}{rl}
R^{*} \cdot W & =R^{*} \cdot \operatorname{Diag} \frac{f}{h} \cdot R \cdot \operatorname{Diag} \frac{c}{q}, \text { de format } m \times m \\
& =\operatorname{Diag} \frac{q}{c} \cdot W^{*} \cdot \operatorname{iag} \frac{h}{f} \cdot R \\
& =\operatorname{Diag} \frac{1}{c} \cdot G^{*} \cdot \operatorname{iag} \frac{1}{g} \cdot G \cdot \operatorname{Diag} \frac{1}{q}
\end{array}\right\}
$$

Remarquons que toutes les équations établies à partir de (2.18) l'ont été indépenda mment des hypothèses relatives à la ventilation, c'est-à-dire qu'on peut les obtenir pour chaque tableau à double entrée $G$ dont les totaux marginaux $g$ et $\Gamma$ peuvent s'écrire sous la forme (1) et (2.1).

Elles n'en sont pas moins tributaires du choix de $T$. Pour un même ensemble de grandeurs $f, h, A, b$ et $q$, chaque approximation $T$, dépendant de $n(s-1)$ paramètres, entraîne un $G$ qui lui-même donne lieu à un $W$ et un $R$. Mais, de plus, toute modification apportée à $q$ se répercutera aussi sur $A$. Aussi, faut-il distinguer le principe de l'existence d'un équilibre entre $f$ et $q$, celui de la ventilation permettant une approximation de la relation d'équilibre, et enfin celui de la stabilité de cette approximation ponctuelle.

Les équations établies sont en quelque sorte des photographies instantanées et déformantes d'un équilibre comptable. L'intérêt majeur de la structure matricielle dégagée est précisément de permettre l'examen des caractères approximatif et instable des relations d'équilibre obtenues. 
Tableau $b$

\begin{tabular}{|c|c|c|c|c|c|}
\hline & \multirow{2}{*}{$\begin{array}{c}\text { Prix } \\
\text { FACTORIEL } \\
h\end{array}$} & \multirow{2}{*}{$\underset{f}{\text { Facteur }}$} & \multirow{2}{*}{$\begin{array}{c}\text { Charge } \\
g\end{array}$} & \multicolumn{2}{|c|}{ Ventilation comptable } \\
\hline & & & & $x$ & \\
\hline Matières premières .... & $\begin{array}{l}\mathrm{F} \\
5\end{array}$ & $310 \mathrm{~kg}$ & $\underset{1550}{\mathbf{F}}$ & $300+\frac{50}{4}+\frac{50}{4,4}$ & $500+$ \\
\hline Salaires .. & 1 & 1700 heures & 1700 & $350+\frac{100}{4}+\frac{150}{4,4}$ & $200+$ \\
\hline Appointements. & 2 & 350 jours & 700 & $200+\frac{100}{4}+\frac{150}{4,4}$ & $100+$ \\
\hline Énergie & 10 & $45 \mathrm{~kW}$ & 450 & $50+\frac{50}{4}$ & $250+$ \\
\hline Matières consommation & 5 & 90 litres & 450 & $50+\frac{50}{4}+\frac{50}{4,4}$ & 200 \\
\hline Amortissements & 10 & 39 jours & 390 & $50+\frac{50}{4}+\frac{40}{4,4}$ & 150 \\
\hline \multicolumn{3}{|l|}{ Coût total } & 5240 & $1000+100+100$ & $1400+$ \\
\hline \multicolumn{4}{|l|}{ Coût total par produit $\Gamma$} & 1200 & \\
\hline \multirow{2}{*}{\multicolumn{4}{|c|}{ Quantité produite $q \ldots \ldots$}} & 100 & \\
\hline & & & Prix de revient complet $c$ & 12 & \\
\hline
\end{tabular}




\section{Exemple}

Sur l'exemple, tableau $a$, en appliquant à chaque frais par nature de chaque section transitoire, les clés de ventilation adoptées, on obtient le tableau $b$ exprimant la matrice de ventilation du coût total $G$. Le tableau a été bordé des vecteurs significatifs du système :

Après calcul de $x_{i j}$ par (2), on obtient

$$
\left.\begin{array}{r}
t=W \cdot q \\
1700 \\
350 \\
90 \\
39
\end{array}\right]=\left[\begin{array}{ccc}
0,6+\frac{2,1}{44} & 1+\frac{2,5}{44} & 0,65+\frac{2,1}{44} \\
3,5+\frac{26}{44} & 2+\frac{32}{44} & 4,5+\frac{26}{44} \\
1+\frac{13}{44} & 0,5+\frac{16}{44} & 0,375+\frac{13}{44} \\
0,05+\frac{0,55}{44} & 0,25+\frac{0,55}{44} & 0,05+\frac{0,55}{44} \\
0,1+\frac{2,1}{44} & 0,4+\frac{2,5}{44} & 0,1+\frac{2,1}{44} \\
0,05+\frac{0,95}{44} & 0,15+\frac{1,11}{44} & 0,05+\frac{0,95}{44}
\end{array}\right] \cdot\left[\begin{array}{l}
100 \\
100 \\
200
\end{array}\right]
$$

tandis qu'après calcul de $r_{i j}$ par (7), on obtient

$$
\left[\begin{array}{l}
100 \\
100 \\
200
\end{array}\right]=\frac{1}{44}\left[\begin{array}{cccccc}
\frac{475}{124} & \frac{15}{17} & \frac{19}{7} & \frac{275}{54} & \frac{325}{108} & \frac{175}{26} \\
\frac{375}{82} & \frac{300}{697} & \frac{380}{287} & \frac{1925}{123} & \frac{1675}{246} & \frac{6425}{533} \\
\frac{1535}{186} & \frac{112}{51} & \frac{59}{21} & \frac{275}{27} & \frac{325}{54} & \frac{175}{13}
\end{array}\right] \cdot\left[\begin{array}{r}
310 \\
1700 \\
350 \\
45 \\
90 \\
39
\end{array}\right]
$$

\section{Modèles linéaires dans l'espace des valeurs}

$\mathrm{Si}$, en général, l'équation (3) n'est pas celle d'un modèle linéaire de production qui postulerait l'indifférence des composantes de $W$ à $q$ et en particulier ramènerait $W$ à $\operatorname{Diag} \frac{1}{h} \cdot A$, par contre, l'équation matricielle du prix de revient (5) est un modèle linéaire. En effet, si par exemple on doublait les prix factoriels, toutes les composantes de $G$ seraient doublées, de même que les prix de revient, tandis que $W$ resterait inchangé. 
Ainsi, pour tout scalaire $\lambda$, on peut écrire (5) sous la forme

$$
\lambda c=W^{*} \cdot \lambda h
$$

De même, tout écart $\Delta h \gtrless 0$ entraîne un écart des charges

$$
\Delta g=h \cdot \operatorname{Diag} f
$$

qui se répercute sur chaque composante de $G$, mais laisse inchangée chaque composante de $W$, de telle sorte qu'au vecteur d'écart des prix factoriels $\Delta h$, correspond un vecteur d'écart des prix de revient complets $\Delta c$ satisfaisant à

$$
\Delta c=W^{*} \cdot \Delta h
$$

Un raisonnement analogue permet d'écrire

et

$$
\left.\begin{array}{rl}
\lambda h & =R \cdot \lambda c \\
\Delta h & =R \cdot \Delta c
\end{array}\right\}
$$

Ainsi, chacun des systèmes peut toujours être étudié dans l'espace des valeurs, soit avec (5) et (11), comme modèle linéaire.

L'équation (5) permet aussi l'étude directe d'une variation proportionnelle des prix factoriels $\pi$ définic par

puisqu'on peut écrire

$$
\pi_{i}=\frac{\Delta h_{i}}{h_{i}} \quad \text { ou } \quad \Delta h=\operatorname{Diag} h \cdot \pi
$$

$$
\Delta c=W^{*} \cdot \operatorname{Diag} h \cdot \pi
$$

Cette propriété à elle seule suffit à montrer l'intérêt pratique de (5). Ainsi, sur l'exemple, une augmentation de $10 \%$ des salaires, les autres facteurs étant inchangés, entraîne une augmentation de prix de revient complet

$$
\Delta c=\left[\begin{array}{l}
0,35+2,6 / 44 \\
0,2+3,2 / 44 \\
0,45+5,2 / 44
\end{array}\right]
$$

soit une augmentation de $0,273 \mathrm{~F}$ du prix du $2^{\mathrm{e}}$ produit $Y$.

\section{Formats particuliers}

Lorsque le format $n \times m$ de $G$ est particulier, les expressions de $W$ et de $R$ méritent d'être distinguées.

Le cas $m=n=1$, e'est-à-dire une production simple à partir d'un seul facteur, entraîne

$$
\begin{aligned}
& g=h f=c q=G \\
& \text { avec } \quad f=\frac{c}{h} q=\$ q \quad \text { et } \quad q=\frac{h}{c} f=r f \\
& \text { d'où } w=\frac{1}{r}
\end{aligned}
$$


En général, $w$ est fonction de $q$, le cas de constance, soit de proportionnalité entre $f$ et $q$, étant particulier.

Le cas $m=1$, c'est-à-dire d'une production simple avec plusieurs facteurs, entraîne

$$
\begin{array}{cc}
g_{i j}=g_{i}=h_{i} f_{i} & \\
\text { avec } & w=\frac{1}{q}\left(f_{i}\right)=\left(w_{i}\right) \quad \text { et } \quad R=\frac{1}{c}\left(h_{i}\right)=\left(r_{i}\right) \\
\text { d'où } & R^{*} \cdot W=\frac{h^{*} \cdot f}{c q}=\sum_{i} r_{i}\left(w_{i}=1\right. \\
W \cdot R^{*}=\frac{1}{h^{*} \cdot f}\left[\begin{array}{ll}
f_{1} h_{1} & f_{1} h_{n} \\
f_{n} h_{1} & f_{n} h_{n}
\end{array}\right]
\end{array}
$$

Les relations (14) sont triviales car satisfaites respectivement par tout couple $(f, h)$ et $(q, c)$.

Ici. le coefficient technique $w_{i}$ et le coefficient de rendement $r_{i}$ ne sont pas inverses l'un de l'autre comme dans le cas d'une production simple. Ils sont par ailleurs bien déterminés.

Il n'est cependant pas rare de trouver une conception différente qui consiste à définir un coefficient à partir de l'autre, soit de poser $\psi_{i} r_{i}=1 \forall i$, façon de procéder incompatible avec (21).

Le cas $n=1$, d'une production multiple à partir d'un seul facteur, soit de produits joints, entraîne une ventilation de coût en général assez arbitraire :

$$
g_{i j}=\Gamma_{j}=c_{j} q_{j}
$$

a vec

$$
W=\frac{1}{h}\left(c_{j}\right)=\left(w_{j}\right) \quad \text { et } \quad R=\frac{1}{f}\left(q_{j}\right)=\left(\boldsymbol{r}_{j}\right)
$$

d'où

$$
R^{*} \cdot W=\frac{q^{*} \cdot c}{h \dot{h}}=\Sigma w_{j} r_{j}=1
$$

et

$$
W \cdot R^{*}=\frac{1}{q^{*} \cdot c}\left[\begin{array}{ll}
c_{1} q_{1} & c_{1} q_{n} \\
c_{n} q_{1} & c_{n} q_{n}
\end{array}\right]
$$

Ici encore, les relations (14) sont triviales. Les coefficients techniques $w_{J}$ et $r_{j}$ ne sont pas inverses l'un de l'autre, mais l'arbitraire de $\Gamma_{j}$ se retrouve dans $w_{j}$ et $c_{j}$, non dans $r_{j}$ puisque $q_{j}$ et $f$ sont déterminés.

Le cas $n=m$ correspond à l'existence de matrices carrées. $f$ et $q$ étant connus, le déterminant associé à $W$ est donc différent de zéro, soit $\operatorname{det} \mathrm{W} \neq 0$, et $W$ possède une inverse, c'est-à-dire que le couple $(f, q)$ satisfaisant à

doit aussi satisfaire à

$$
\begin{gathered}
f=W \cdot q \\
q=W-1 \cdot f \\
q=R^{*} \cdot f
\end{gathered}
$$$$
\text { ainsi qu'à }
$$ 
ce qui n'entraîne cependant pas nécessairement

$$
W^{-1}=R^{*}
$$

Cette égalité ne correspond qu'à un cas particulier pour lequel on a également $R^{*-1}=W$, c'est-à-dire que les deux systèmes se confondent.

Avec (23), la matrice des coefficients de rendement s'obtient alors directement à partir de celle des coefficients techniques et inversement, sans référence aux autres vecteurs du système explicités dans (13) et sans ambiguïté : aucune composante n'est négative.

(23) implique pour $G$

$$
\begin{aligned}
\operatorname{Diag} q \cdot G^{-1} \cdot & \operatorname{Diag} h=\operatorname{Diag} \frac{1}{c} \cdot G^{*} \cdot \operatorname{Diag} \frac{1}{f} \\
G^{-1} & =\operatorname{Diag} \frac{1}{\Gamma} \cdot G^{*} \cdot \operatorname{Diag} \frac{1}{g} \\
& =\left[\frac{g_{i j}}{g_{i} \Gamma_{j}}\right]^{*}
\end{aligned}
$$

\section{4) PRINCIPE DE FIXITE}

Si $\varphi$ est le vecteur prix de vente de $q$, le calcul de $c$ permet celui d'un vecteur bénéfice unitaire $(\varphi-c)$, indicateur d'orientation de la production. En effet, en admettant que le calcul de $c$ soit suffisamment correct nonobstant l'arbitraire de la ventilation, la classification des $\left(v_{j}-c_{j}\right)$ par ordre de grandeur décroissante donne l'échelle de préférence de l'accroissement de production le plus profitable.

Ainsi, partant d'une matrice de ventilation $G$ associée à la production $q$, le calcul de $c$ entraîne l'orientation de la production vers le niveau $q^{(1)}$, qui conduit à une matrice $G^{(1)}$ et aux prix de revient $c^{(1)}$ qui, à leur tour, orientent vers $q^{(2)}$, etc.

Cette adaptation successive influencée par la ventilation adoptée permet d'observer la fixité de certaines composantes de $G$. Plus précisément, dans

$$
g_{i}=\sum_{j} g_{i j}=\sum_{j} a_{i j}+b_{i}
$$

certaines parties sont ou peuvent être considérées comme fixes, notamment les $b_{i}$.

Le principe de fixité des charges transitoires se substituant à celui de leur ventilation, la matrice $G$ se ramène à un cas simple d'affectation. Au calcul d'un prix de revient complet, on substitue alors celui d'un prix de revient partiel $\gamma$ qui indique l'accroissement de coût entraîné par l'accroissement d'une unité de produit, soit l'accroissement marginal de coût. Si $\gamma$ est constant quel que soit le niveau de production considéré, le modèle est linéaire et la comptabilité est dite marginale, mais le cas reste 
assez exceptionnel car, le plus souvent, $\gamma$ varie avec le niveau de production. A la comptabilité marginale, se substitue dès lors la comptabilité directe. D'autre part, le choix des facteurs explicatifs et celui de la zone de production représentée influencent la nature du prix de revient partiel. Sur l'exemple, en ne retenant que les facteurs à variation proportionnelle, on obtient le tableau $c$ qui permet le calcul des marges brutes unitaires entre les prix de vente considérés et les prix de revient marginaux :

\section{TABLEAU $c$}

\begin{tabular}{|c|c|c|c|}
\hline & \multicolumn{3}{|c|}{ Produits } \\
\hline & $X$ & $Y$ & $Z$ \\
\hline Matières. & $300 \mathrm{~F}$ & $500 \mathrm{~F}$ & $650 \mathrm{~F}$ \\
\hline Salaires .. & 350 & 200 & 900 \\
\hline Énergie ... & 50 & 250 & 100 \\
\hline Total frais proport. . . & 700 & 950 & 1650 \\
\hline Nombre d'unités............... & $100 X$ & $100 Y$ & $200 Z$ \\
\hline Prix de revient marginal . . . . . . . . & $7 \mathrm{~F}$ & $9,50 \mathrm{~F}$ & $8,25 \mathrm{~F}$ \\
\hline Prix de vente $\ldots \ldots \ldots \ldots \ldots \ldots \ldots$ & 15 & 19,00 & 16,00 \\
\hline Marge brute unitaire $\ldots \ldots \ldots \ldots$ & 8 & 9,50 & 7,75 \\
\hline
\end{tabular}

TABLEAU $d$

\begin{tabular}{|c|c|c|c|}
\hline & \multicolumn{3}{|c|}{ Produits } \\
\hline & $X$ & $Y$ & $Z$ \\
\hline Matières premières. $\ldots \ldots \ldots \ldots \ldots$ & $300 \mathrm{~F}$ & $500 \mathrm{~F}$ & $650 \mathrm{~F}$ \\
\hline Salaires $\ldots \ldots \ldots \ldots \ldots \ldots \ldots \ldots$ & 350 & 200 & 900 \\
\hline Appointements. $\ldots \ldots \ldots \ldots \ldots \ldots$ & 200 & 100 & 150 \\
\hline Énergie $\ldots \ldots \ldots \ldots \ldots$ & 50 & 250 & 100 \\
\hline Matières consommation........... & 50 & 200 & 100 \\
\hline Total frais variables.............. & 950 & 1250 & 1900 \\
\hline Nombre d'unités........... & $100 X$ & $100 Y$ & $200 Z$ \\
\hline Prix de revient variable & $9,5 \mathrm{~F}$ & $12,5 \mathrm{~F}$ & $9,5 \mathrm{~F}$ \\
\hline Prix de vente $\ldots \ldots \ldots \ldots \ldots \ldots$ & 15,0 & 19,0 & 16,0 \\
\hline $\begin{array}{l}\text { Marge brute unitaire du prix de } \\
\text { revient variable } \ldots \ldots \ldots \ldots \ldots \ldots \ldots\end{array}$ & 5,5 & 6,5 & 6,5 \\
\hline
\end{tabular}


Ces marges brutes unitaires indiquent l'orientation à donner à la promotion de la production, soit

$$
Y>X>Z
$$

$>$ signifiant "est préférable à ".

Par contre, en considérant les facteurs variables, on obtient le tableau $d$, d'où l'échelle de promotion

$$
Y=Z>X
$$

Mais l'orientation de la production définie par la marge bénéficiaire la plus profitable n'est pas nécessairement la même que celle découlant de la marge bénéficiaire la plus rentable, celle qui couvre le plus rapidement les frais fixes engagés, quelle que soit leur importance. On le voit sur le tableau e résumant les diverses voies d'évaluation de la hiérarchie des promotions :

\begin{tabular}{|c|c|c|c|c|c|c|c|c|c|c|}
\hline \multirow[b]{2}{*}{ Produit } & \multirow{2}{*}{$\begin{array}{c}\text { PRIX } \\
\text { DE } \\
\text { VENTE } \\
\rho\end{array}$} & \multicolumn{3}{|c|}{ P. r. COMPLET $c$} & \multicolumn{3}{|c|}{ P. r. MARGinal $m$} & \multicolumn{3}{|c|}{ P. r. DIRECT $\gamma$} \\
\hline & & $c$ & $v-c$ & $\frac{v-c}{v}$ & $m$ & $\varphi-m$ & $\frac{v-m}{\varphi}$ & $\gamma$ & $0-Y$ & $\frac{\rho-\gamma}{\nu}$ \\
\hline & $\mathbf{F}$ & $\mathrm{F}$ & $\mathrm{F}$ & $\%$ & $\mathrm{~F}$ & F & $\%$ & $\mathrm{~F}$ & F & $\%$ \\
\hline $\boldsymbol{X}$ & 15,0 & 12,0 & 3,0 & 20 & 7,0 & 8,0 & 53,3 & 9,5 & 5,5 & 46,7 \\
\hline$Y$ & 19,0 & 16,4 & 2,6 & 13,7 & 9,5 & 9,5 & 50 & 12,5 & 6,5 & 34,4 \\
\hline$Z$ & 16,0 & 12,0 & 4,0 & 25 & 8,25 & 7,75 & 47,8 & 9,5 & 6,5 & 40,7 \\
\hline Hiérarchie & & & & & & $Y$ & $X$ & & $X$ & $X$ \\
\hline de & & & & & & $X$ & $Y$ & & $Y=Z$ & $Z$ \\
\hline promotion & & & & & & $Z$ & $Z$ & & & $Y$ \\
\hline
\end{tabular}

TABLEAU $e$

La diversité des solutions suffit à montrer la précarité du marginalisme.

\section{5) OPTIMISATION MARGINALISTE}

Les difficultés de définition des marges bénéficiaires, soit celles de distinguer entre les frais indirects, fixes et de structure, ces derniers couvrant à leur tour soit des frais de période soit des frais de programme selon les opportunités, ne reflètent pas de simples querelles académiques de terminologie. La fixité de certains frais serait aussi illusoire que la pertinence de leur ventilation.

En résumé, la comptabilité des prix de revient complets, basée sur le principe de ventilation, et les comptabilités "marginalistes " basées sur 
le principe de fixité de certains frais permettent seulement des approximations de la structure des coûts.

Or, à propos du calcul du prix de revient complet, nous avons établi les équations fondamentales

$$
\left\{\begin{array} { l } 
{ f = W \cdot q } \\
{ c = W ^ { * } \cdot h }
\end{array} \quad \text { et } \quad \left\{\begin{array}{l}
q=R^{*} \cdot f \\
h=R \cdot c
\end{array}\right.\right.
$$

liées à $G$, tableau comptable à double entrée : charges $\times$ coûts, par

$$
W=\operatorname{Diag} \frac{1}{h} \cdot G \cdot \operatorname{Diag} \frac{1}{q} \quad \text { et } \quad R=\operatorname{Diag} \frac{1}{f} \cdot G \cdot \operatorname{Diag} \frac{1}{c}
$$

en soulignant que de telles équations pouvaient être écrites pour tout tableau comptable $G$, c'est-à-dire que (1), (2) et (3) sont également possibles en comptabilité marginaliste, mais cette fois non seulement (2) expriment des modèles linéaires sur l'espace des valeurs, mais en outre (1), sont valables sur des ensembles de $f$ et de $q$, soit $\mathfrak{F}$ et $Q$ non réduits à un point. (1) devient dès lors une transformation sur l'espace des biens valable pour un sous-espace. Ce sous-espace conditionne le traitement comptable et ses conclusions éventuelles. Si ces conclusions sont des propriétés nécessaires et suffisantes de ce sous-espace, elles seront insuffisantes pour une comptabilité valable dans un sous-espace inclus, mais néanmoins nécessaires, d'où l'idée d'une approche dynamique en supposant d'abord (1) modèle linéaire. Dès lors, plusieurs combinaisons $(f, q)$ étant possibles sur (1), on peut rechercher la ou les plus intéressantes par rapport à un critère d'efficacité, c'est-à-dire qu'on peut chercher à optimiser le système de production ou celui de consommation par rapport à un critère choisi.

De nombreuses applications de programmation linéaire sont des exemples de telles optimisations marginalistes.

Ainsi, à partir du tableau $c$, l'équation fondamentale de production sur le sous-espace des biens $f=W \cdot q$ étant

$$
\left[\begin{array}{r}
290 \\
1450 \\
40
\end{array}\right]=\left[\begin{array}{lll}
0,6 & 1 & 0,65 \\
3,5 & 2 & 4,5 \\
0,05 & 0,25 & 0,05
\end{array}\right] \cdot\left[\begin{array}{l}
100 \\
100 \\
200
\end{array}\right]
$$

on peut rechercher le vecteur production $q=(x, y, z)$ maximisant la marge brute totale, soit

$$
\operatorname{Max}(8 x+9,5 y+7,75 z)
$$

pour une consommation factorielle limitée par le vecteur $f$ considéré, c'est-à-dire si l'équation fondamentale est valable pour tout $f$ inférieur à ce vecteur, pour 


$$
\begin{aligned}
290 & \geqslant 0,6 x+y \quad+0,65 z \\
1450 & \geqslant 3,5 x+2 y+4,5 z \\
40 & \geqslant 0,05 x+0,25 y+0,05 z
\end{aligned}
$$

avec

$$
x \geqslant 0 \quad y \geqq 0 \quad z \geqq 0
$$

Il s'agit bien dès lors d'un cas particulier du programme linéaire définissant une production utilisant des ressources données et maximisant la marge brute totale

$$
\left.\begin{array}{l}
f \geqslant W \cdot q \\
q \geqq 0 \\
\operatorname{Max} m^{*} \cdot q
\end{array}\right\}
$$

où $m$ est le vecteur marge brute unitaire.

La solution d'équilibre tirée par la comptabilité rapporte la marge brute totale $3300 \mathrm{~F}$, mais la solution

$$
x=378 \quad y=63 \text { et } z=0
$$

rapporterait $3622,5 \mathrm{~F}$.

C'est la solution en entier la plus proche de l'optimum.

Cet exemple prouve que le tableau comptable dont on part, tableau d'affectation ou de ventilation, exprime un équilibre, mais pas nécessairement un optimum.

En outre, l'exemple révèle que l'orientation donnée par la comptabilité marginale, soit $Y>X>Z$, ne concorde pas nécessairement avec l'orientation qu'exigerait la solution optimale (mais ici avec l'orientation donnée par la marge brute proportionnelle, soit $X>Y>Z$ ).

Si l'équilibre comptable correspondait à l'optimum, c'est-à-dire si sur le sous-espace de (4)

avec

$$
\left.\begin{array}{rl}
\operatorname{Max} m^{*} \cdot q & =m^{*} \cdot q^{0} \\
f^{0} & =W q^{0} \\
q^{0} & \geqq 0
\end{array}\right\}
$$

et

le problème d'orientation serait celui d'élargir les contraintes, soit d'exprimer $\Delta f^{0}$ en fonction de $\Delta q^{0}$. Or

$$
\Delta f^{0} \neq W \cdot \Delta q^{0}
$$

sinon $W$ resterait la matrice des coefficients techniques de production associée au nouveau sous-espace considéré, c'est-à-dire que le programme (4) aurait été mal posé quant aux contraintes.

Cette remarque attire l'attention sur le caractère extrémiste de l'optimisation marginaliste et sur l'intérêt d'étudier $W$ précisément à l'endroit de l'espace des biens où $W$ change, c'est-à-dire paradoxalement là où la comptabilité marginaliste est défaillante. 


\section{6) PRincipe DU PARTAge Des Responsabilites}

Lorsque l'entreprise occupe sur le marché une position assurée, l'approximation inhérente à la ventilation ou au marginalisme ne peut lui être fatale à court terme, mais il en va autrement lorsque l'entreprise vivote dans la zone de son point mort, e'est-à-dire de son équilibre recettes-dépenses car la répétition de pertes faibles non seulement réduit les ressources de l'entreprise, mais, de plus, l'empêche de suivre la progression des autres. Aussi, les écarts sur la position d'équilibre sont-ils plus importants que le niveau absolu du prix de revient complet ou la tendance immédiate du prix de revient partiel.

Cette position d'équilibre peut, à son tour, être interprétée très diversement. Elle peut s'exprimer, comme le professe l'École d'Amsterdam, au travers d'un prix d'utilisation permettant une vente des produits avec un bénéfice normal. La comptabilité des coûts standards, dérivant euxmêmes de prix standards, d'une qualité standard, en quantité standard et plus généralement encore la comptabilité de coûts préétablis intégrés dans un modèle théorique de fonctionnement de l'entreprise, valent au contraire dans la mesure où elles reflètent effectivement un état de l'entreprise assurant sa viabilité et une orientation à donner à l'entreprise dans diverses situations du marché.

La référence étant bien connue et admise par les niveaux exécutants, le contrôle de la réalisation visée dégagera des écarts dosant en quelque sorte les responsabilités des exécutants. Ce partage des responsabilités, même s'il se fait dans l'esprit d'éclairer la voie de redressement ultérieur, et non de sanctionner des erreurs commises, se heurte cependant à son tour à des contradictions internes : l'amélioration d'un secteur peut se faire au détriment d'un autre, diverses situations exigeant des arbitrages de la direction. La résorption des écarts peut échapper aux adaptations tactiques de l'exécution, mais exiger une action stratégique affectant la structure interne du système.

Il semble néanmoins que le point litigieux, celui qui neutralise la validité des divers principes envisagés tour à tour : ventilation, fixité, partage des responsabilités, soit précisément cette évolution permanente de la structure et que l'effort d'analyse doive permettre de la cerner et de la prévoir.

\section{7) ANALYSE DES ECARTS}

La comptabilité à prix standard ou la comptabilité budgétaire impliquent l'analyse des écarts entre le réel et la référence admise : standard, budget ou modèle théorique. Néanmoins, cette analyse est délicate ; elle peut notamment prendre plusieurs formes. Ainsi, pour une production simple, l'écart du coût réel $C_{T}=c q$ sur le coût standard (ou budgété ou théorique) $\hat{C}_{T}=\hat{c} \hat{q}$, soit

$$
\Delta C_{T}=C_{T}-\hat{C}_{T}=c q-\hat{c} \hat{q}
$$


peut s'écrire

ou

$$
\left.\begin{array}{l}
\Delta C_{T}=c(q-\hat{q})+\hat{q}(c-\hat{c}) \\
\Delta C_{T}=\hat{c}(q-\hat{q})+q(c-\hat{c})
\end{array}\right\}
$$

d'où l'on peut déduire pour tous scalaires $\lambda$ et $\mu$ tels que $\lambda+\mu=1$

$$
\Delta C_{r}=(\lambda c+\mu \hat{c}) \Delta q+(\lambda \hat{q}+\mu q) \Delta c
$$

Il en résulte que si l'écart de coût correspond à un écart de prix et à un écart de quantité, la décomposition entre ces deux causes reste indéterminée.

Si on exprime l'écart de coût par rapport à ses composantes théoriques en prix et en quantité, c'est-à-dire si on écrit

on a par contre

$$
\Delta C_{T}=(\hat{c}+\Delta c)(\hat{q}+\Delta q)--\hat{c} \hat{q}
$$

$$
\Delta C_{T}=\hat{c} \Delta q+\hat{q} \Delta c+\Delta q \Delta c
$$

Dans (4), l'indétermination de (3) paraît levée et on vérifie aisément que

$$
\Delta q \Delta c=(\lambda c+\mu \hat{c}) \Delta q-\hat{c} \Delta q+(\lambda \hat{q}+\mu q) \Delta c-\hat{q} \Delta c
$$

L'indétermination de l'analyse (3) réside donc dans un effet de covariance des quantités et des prix, effet de second ordre négligeable pour de petits écarts, comme en analyse infinitésimale, mais qu'il serait dangereux d'ignorer systématiquement dans l'analyse des écarts.

L'analyse précédente est générale : on peut la répéter sur les équations fondamentales de production et de consommation. Ainsi, à partir d'un système de référence caractérisé par le tableau comptable charges $\times$ couts $\hat{G}$ et du système réel caractérisé par le tableau de même forme $G$, on peut notamment écrire les équations fondamentales de consommation

$$
f=W \cdot q \quad \text { et } \quad \hat{f}=\hat{W} \cdot \hat{q}
$$

d'où, comme (3) ci-dessus,

$$
\Delta f=f-\hat{f}=(\lambda W+\mu \hat{W}) \Delta q+\Delta W \cdot(\lambda \hat{q}+\mu q)
$$

où $\lambda$ et $\mu$ sont des scalaires réels tels que $\lambda+\mu=1$, mais aussi comme (4) ci-dessus

soit

$$
\Delta f=\hat{W} \cdot \Delta q+\Delta W \cdot \hat{q}+\Delta W \cdot \Delta q
$$

$$
\Delta f=\hat{W} \cdot \Delta q+\Delta W \cdot q
$$

montrant que l'écart de consommation $\Delta f$ ne résulte pas seulement d'un simple effet d'écart de production (ce que nous avions déjà observé en 2.6), mais qu'il résulte aussi d'un effet d'écart de structure. Ainsi, $\Delta W \cdot q$ est l'effet de structure pour le niveau de production atteint. 
L'analyse des écarts, tout en restant statique dans son principe, fait bien ressortir la nécessité d'exprimer la façon dont la structure du système identifiée par $W$ varie avec le niveau de production $q$, soit la nécessité d'étudier la dynamique du système. Aux équations fondamentales de consommation et de production, se substituent des équations dynamiques telles que

et

$$
\left.\begin{array}{l}
f=\hat{W} \cdot \hat{q}+\hat{W} \cdot \Delta q+\Delta W \cdot q \\
q=\hat{R}^{*} \cdot f+\hat{R}^{*} \cdot \Delta f+\Delta R^{*} \cdot f
\end{array}\right\}
$$

La mesure intra-comptable du flux des écarts permet d'ailleurs de cerner cet effet de structure, embué toutefois de l'incidence des variations des prix factoriels.

En effet, le graphe comptable :

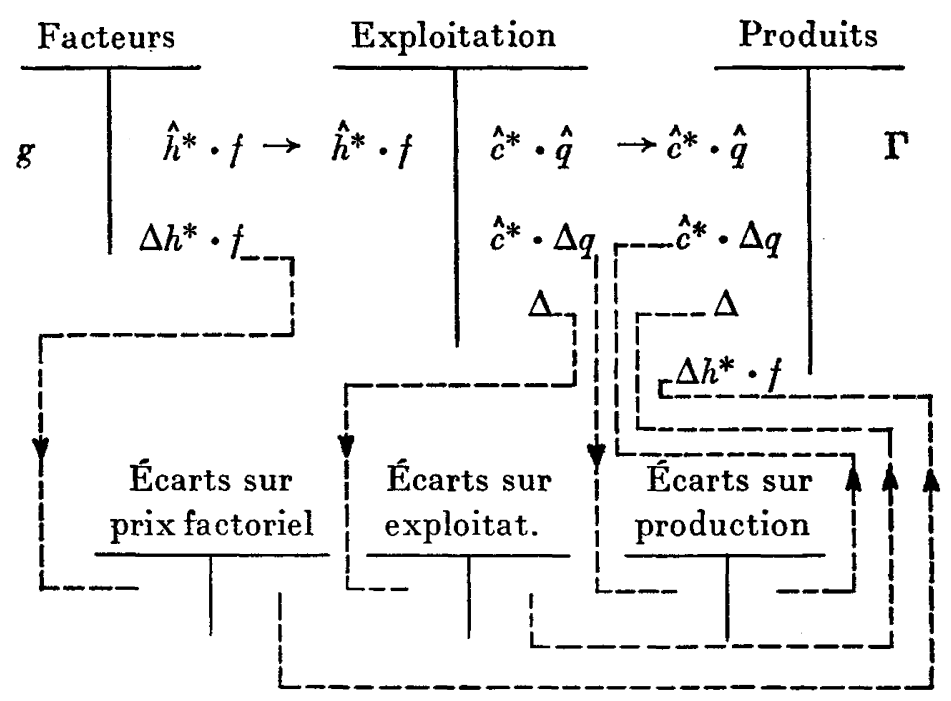

expliqué par les relations

$$
\begin{gathered}
g=h^{*} \cdot f=(\hat{h}+\Delta h)^{*} \cdot f \\
\hat{h}^{*} \cdot f=\hat{h}^{*} \cdot W \cdot q=\hat{h}^{*} \cdot \hat{W} \cdot q+\hat{h}^{*} \cdot \Delta W \cdot q \\
=\hat{c}^{*} \cdot q+\hat{h}^{*} \cdot \Delta W \cdot q \\
=\hat{c}^{*} \cdot \hat{q}+\hat{c}^{*} \cdot \Delta q+\Delta
\end{gathered}
$$

et $\quad \Gamma=g$

explicite $\Delta$, dit écart sur exploitation qui peut s'écrire

$$
\Delta=\hat{h}^{*} \cdot \Delta W \cdot q
$$

soit l'effet de structure au niveau $q$, effet valorisé aux prix factoriels de référence $\hat{h} ; \Delta$ est donc la composante dynamique du coût. 
On peut encore exprimer $\Delta$ par

$$
\Delta=\hat{h}^{*} \cdot f-\hat{c}^{*} \cdot q
$$

donnant l'écart entre charges et coûts, valorisés l'un et l'autre aux prix de référence, ou par

$$
\Delta=\hat{h}^{*} \cdot \Delta f-\hat{c}^{*} \cdot \Delta q
$$

puisque par construction

$$
\hat{g}=\hat{h}^{*} \cdot \hat{f}=\hat{c}^{*} \cdot \hat{q}=\hat{\Gamma}
$$

(10) montre que si

$$
f=\hat{f} \text { et } q=\hat{q}, \quad \text { alors } \quad \Delta=0
$$

c'est-à-dire que si l'exploitation réalise exactement le modèle, tout écart sur résultat sera dû à un écart de prix factoriel. Si la direction générale fixe le modèle et si le service d'exploitation le réalise, la responsabilité de l'écart total revient donc au service d'achat, soit à la conjoncture, c'est-à-dire à personne.

Cette conclusion un peu trop commode rappelle les difficultés du principe de partage des responsabilités.

(8) et (9) montrent aussi que le coût total pour un vecteur de prix factoriel fixé, soit $\hat{h}^{*} \cdot f$, se compose d'une partie fixe : le coût de référence, d'une partie fonction linéaire de la variation de production $\hat{c}^{*} \cdot \Delta q$, et d'une partie dynamique : l'écart sur exploitation, fonction plus complexe du niveau de production, soit

$$
\hat{h}^{*} \cdot f=\hat{\Gamma}+\hat{c}^{*} \cdot \Delta q+\hat{h}^{*} \cdot \Delta W \cdot q
$$

tandis que (10) présente le même coût de référence comme la somme d'une fonction linéaire et d'une fonction plus complexe du niveau de production, soit

$$
\hat{h}^{*} \cdot f=\hat{c}^{*} \cdot q+\hat{h}^{*} \cdot \Delta \mathrm{W} \cdot q
$$

montrant que les prix de revient complets correspondant aux prix factoriels de référence $\hat{h}$ seront

$$
\hat{c}^{*}+\hat{h}^{*} \cdot \Delta W
$$

c'est-à-dire seront composés d'une partie fixe : le prix de revient de référence, et d'une partie fonction de l'écart de structure : $\Delta W$.

Nonobstant l'intérêt de l'évaluation de (9), écart sur exploitation, soit de (6), effet de structure pour le niveau de production atteint, il convient d'observer qu'il s'agit d'une évaluation conditionnée par le choix de référence $(5)$ car $\Delta f$ comme $\Delta W$ comme $\Delta$ restent fonctions de cette référence. Ainsi, l'analyse des écarts reste-t-elle d'intérêt local, c'est-à-dire limité à une zone de production restreinte. 


\section{8) STRUCTURE ADDITIVE}

\section{Charge variable non linéaire maximale}

Ponctuelle quand elle se veut complète, marginale quand elle se veut dynamique, locale quand elle se veut prévisionnelle, la comptabilité réclame cependant dans ses diverses adaptations une structure additive lorsque ses écritures sont isométriques, cas traditionnel, ou plus généralement une structure vectorielle parfaitement adaptée aux moyens actuels du traitement de l'information.

Cette structure, implicite dans chacun des principes de ventilation, de fixité et de responsabilité, explique le traitement auquel la matrice $G$ des charges $\times$ coûts peut être soumise quel que soit le système comptable adopté. A l'additivité d'une charge d'une nature spécifiée concourant à plusieurs produits et à l'additivité des différentes charges exprimées dans une même unité concourant à un même produit, exploitées toutes deux sur la matrice $G$, il convient encore d'adjoindre l'additivité de chaque composante $g_{i j}$ par rapport aux accroissements de production, soit le caractère cumulatif de ces composantes. Bref, la propriété

$$
q>\hat{q} \text { implique } g_{i j}(q) \geqslant g_{i j}(\hat{q})
$$

Ainsi, chaque composante $g_{i j}(q)$ est par nature une fonction numérique non négative croissante de chaque composante de $q$, et même en général strictement croissante car, toutes choses étant égales par ailleurs, il en coûte plus pour produire davantage.

La croissance de $g_{i j}(q)$ est éventuellement émaillée de discontinuités, à savoir : charges fixes à l'origine $g_{i j}(0) \geqslant 0$, charges semi-fixes qui sont des sauts de discontinuité de $1^{\text {re }}$ espèce intervenant aux goulots de production, et changements brusques d'allure des charges marginales correspondant à des points de rebroussement. Ces charges marginales ont cependant une valeur minimale : les charges directement proportionnelles $g_{i j}^{p} \geqslant 0$,

soit

$$
\frac{\partial g_{i}(q)}{\partial q_{j}} \geqslant g_{i j}^{p}
$$

Dès lors, on peut écrire pour toute charge $i$ :

$$
g_{i}(q)=g_{i}(0)+\sum_{j=1}^{n} g_{i j}^{p} q_{j}+g_{i}^{v}(q)
$$

où $g_{i}(0)$ est la charge fixe $\grave{a}$ l'origine sans ventilation et $g_{i}^{v}(q)$ le résidu couvrant la partie variable non linéaire maximale de la charge. Ce résidu reste fonction numérique non négative croissante de $q$, regroupe pour $q>0$ les discontinuités et rebroussements et donne la caractéristique de la structure évolutive du système. 
Si $q^{i}$ est une solution extrémale de (2), et il en existe au moins une par construction, elle satisfait aussi et réciproquement aux $n$ conditions

d'où encore

$$
\frac{\partial g_{i}^{v}\left(q^{i}\right)}{\partial q_{j}}=0 \quad \forall l
$$

tandis qu'en dehors du cas d'égalité

$$
g_{i j}\left(q^{i}\right)-g_{i j}(0)=\sum_{j=1}^{n} g_{i j}^{p} q_{j}^{i}
$$

$$
g_{i j}(q)-g_{i j}(0)>\sum_{j=1}^{n} g_{i j}^{p} q_{j}
$$

\section{Expression minimale de la matrice de Walras}

Si on considère le vecteur des charges, on écrira de même

$$
g(q)=g(0)+G^{p} \cdot q+g^{v}(q)
$$

où $g(0) \supseteqq 0$ est le vecteur des charges fixes d'origine

$G^{p}=\left[g_{i j}^{p}\right] \geqslant 0$ la matrice des charges $i$ directement proportionnelles par unité produite $j$ dans $Q$, ensemble des $q$,

$g_{i j}^{p}$ étant la charge $i$ réclamée par la production d'une unité de $j$, valeur minimale vérifiée au moins en un point $q^{i}$ définie par (4).

$g^{v}(q) \supseteqq 0$ le vecteur des charges variables non linéaires

(5) et (6) conduisent à

$$
g(q)-g(0) \geqq G^{p} \cdot q \quad \forall q \in Q
$$

l'égalité étant obtenue dans le cas où les solutions extrémales de (4) se recouvrent, c'est-à-dire où les $n$ conditions

admettent une solution $q$.

$$
\frac{\partial g^{v}}{\partial q}=0
$$

L'équation (3) étant indifférente aux prix factoriels $h_{i}$, il vient

et aussi à partir de (7)

$$
f_{i}(q)=f_{i}(0)+\sum_{j=1}^{n} f_{i j}^{p} q_{j}+f_{i}^{v}(q)
$$

$$
f(q)=f(0)+W^{p} \cdot q+f^{v}(q)
$$

les symboles ayant la même signification que ci-dessus.

$$
W^{p}=\left[f_{i j}^{p}\right]=\left[\frac{g_{i j}^{p}}{h_{i}}\right] \text { est à son tour l'expression minimale de la }
$$

matrice $W$ des coefficients techniques de production, la relation (8) s'écrivant

$$
W \geqslant W^{p}
$$


Sur l'exemple, au paragraphe $5, W^{p}$ est en fait la matrice considérée. $\mathrm{Si}$, en comptabilité prévisionnelle, on peut écrire

$$
\Delta f=f-\hat{f}=W^{p} \cdot \Delta q+f^{\nu}(q)-f^{\nu}(\hat{q})
$$

il ne faut cependant pas confondre cette décomposition avec celle de l'équation 6, paragraphe 7. On constate d'ailleurs que l'effet de structure pour le niveau de production $q$, soit $\Delta W \cdot q$, est en général sous-estimé puisque par (12)

$$
\hat{W} \geqslant W^{p} \text {. }
$$

\section{Principe d'une gestion par ratio contrôlé}

En procédant comme au paragraphe 2, on déduira pour le coût total

$$
C_{T}(q)=e^{*} \cdot g=C_{T}(0)+\sum_{j=1}^{n} p_{j} q_{j}+C_{T}^{v}(q)
$$

où $C_{T}(0)=e^{*} \cdot g(0)$ est le coût fixe à l'origine

$C_{\mathrm{T}}^{v}(q)=e^{*} \cdot \mathrm{g}^{\mathrm{v}}(q)$ le coût variable non linéaire

et $p_{j}=\sum_{i} g_{i j}^{p}=\sum_{i} h_{i} f_{i j}^{p}$ le prix de resient directement proportionnel du produit $j$.

Ce prix est au plus égal au prix de revient marginal du $j^{\text {ième }}$ produit, qu'il atteint quand (9) a une solution.

Ainsi, la décomposition (3) des charges se conserve sur les coûts : l'additivité est done invariante.

Cette propriété a été exploitée en premier lieu par la gestion marginale transformant (14) en

$$
C_{T}(q)=F_{0}+\sum_{j} m_{j} q_{j}
$$

avec $\quad m_{j}=\sum_{i} \frac{\partial g_{i}}{\partial q_{j}} \quad$ et $\quad F_{0}=C_{T}(0)+\left[C_{T}^{v}(q)-m^{*} \cdot q\right]$

Nous avons montré le danger de cette formulation voilant l'indétermination du système par une déformation des frais fixes; nous avons aussi relevé le risque de croire au sérieux de l'optimisation marginaliste que cette formulation suscite et ce risque est d'autant plus accusé que d'excellents auteurs, ainsi Mattessich et Ijiri, ont en quelque sorte inséré le recours systématique à la programmation linéaire dans leur modèle comptable. Certes, la programmation paramétrée réduirait partiellement l'extrémisme des solutions éventuelles, mais il paraît alors plus expédient, comme l'ont préconisé Holt, Modigliani, Muth et Simon, de faire un usage systématisé d'une expression quadratique des $g^{v}(q)$.

L'intérêt théorique et pratique de cette forme quadratique est suffisamment prouvé par l'étude des séries économiques et le succès de la 
formule de Wilson. Elle n'en constitue pas moins une astuce pour introduire la possibilité de définir un optimum, mais un optimum scolastique nonobstant la présomption de l'existence d'une production optimale dans les industries à coût concave, optimum de circonstance qui changerait d'ailleurs en adoptant une autre formulation polynomiale des charges serrant la réalité de plus près. Ces formulations polynomiales des charges restant additives, plusieurs études extra-comptables de R.O. sont allées dans ce sens, mais il s'agissait alors de fixer des modèles spécifiques.

On peut au contraire couvrir un champ d'application beaucoup plus vaste et plus réaliste en considérant les cas satisfaisant à la condition d'asservissement

$$
\begin{array}{ll}
g^{\nu}(q) \leqslant \lambda g(q) \quad \text { avec } \quad & 0<\lambda<1 \\
& \forall q \in Q
\end{array}
$$

cas pour lequel la charge variable non linéaire ne dépasse pas un pourcentage de la charge totale. Ce cas permet d'accroître ou de diversifier la production en restant assuré que le coût total demeure inférieur à une limite linéaire car (16) est équivalente à

$$
\begin{aligned}
g(q) & \leqslant \frac{1}{1-\lambda}\left[g(0)+G^{p} \cdot q\right] \\
& \leqslant \frac{1}{1-\lambda} \min g(q)
\end{aligned}
$$

où $g(0)+G^{p} \cdot q$ est la charge minimale possible, en général facile à déterminer. L'erreur d'estimation que l'on commettrait sur son niveau se répercuterait simplement sur $\lambda$ sans mettre en cause le principe du contrôle ni la possibilité d'écrire dans les mêmes conditions

$$
\begin{aligned}
C_{T}(q) & \leqslant \frac{1}{1-\lambda}\left[C_{T}(0)+p^{*} \cdot q\right] \\
& \leqslant \frac{1}{1-\lambda} \min C_{T}(q)
\end{aligned}
$$

De plus, $\varphi$ étant le prix de vente, on peut choisir $\lambda$ tel que

$$
\frac{1}{1-\lambda} \min C_{T}(q) \leqslant v \cdot q
$$

et surveiller que le ratio du coût total au coût total minimal pour la production envisagée reste inférieur au ratio du chiffre d'affaires de cette production à son coût total minimum.

On s'assure ainsi que le bénéfice existe, même s'il reste indéterminé a priori, cette indétermination étant d'ailleurs une tare générale des modèles comptables.

Une telle gestion par ratio contrôlé, basée sur la propriété d'invariance de structure des charges et des coûts et sur le plafonnement des 
EQUations Caracteristiques De Comptabilite analytique 105

composantes variables non-linéaires, échappe aux faux problèmes de ventilation, de fixité et de responsabilité. Elle est par ailleurs d'application aisée et les moyens actuels de traitement permettent une mise à jour constante du paramètre $\lambda$. Ce ratio contrôlé pourrait rappeler le coefficient de frais généraux de la comptabilité complète, mais il est inutile de souligner les différences conceptuelles et pratiques entre la limite contrôlée d'une proportion du coût linéaire minimal et l'évaluation relative d'une partie mal définie du coût total.

Cette gestion par ratio contrôlé peut être perfectionnée sous divers aspects et, en particulier, la précision du système sera améliorée en appliquant (16) à chaque charge $g_{i}$ avec un paramètre $\lambda_{i}$ adéquat conduisant à une relation (18) pondérée selon les $g_{i}$, donc adaptable à l'évolution des prix factoriels.

\section{OUVRAGES CITES}

J. Parenteau et $\mathrm{Cl}$. Charmont, Calcul des prix de revient, Comptabilité industrielle, C.E.G.O.S., Paris, $4^{\mathrm{e}}$ édit., 1952.

Robert Henon, L'économétrie au service de l'entreprise, Gauthier-Villars, Paris, 1964.

Louis Perridon, Le calcul du profit par l'École d'Amsterdam. Publication de I'Institut Technique des Salaires, Paris, 1951.

Richard Matressich, Accounting and Analytical Methods. Richard D. Irwin, Inc., Homewood, Illinois, 1964.

Yuji IJIRI, Management Goals and Accounting for Control. North Holland Publishing Cy, Amsterdam, 1965.

Holt, Modigliani, Muth et Simon, Planification de la production, des stocks et de l'emploi. Traduit de l'anglais. Dunod, Paris, 1964. 